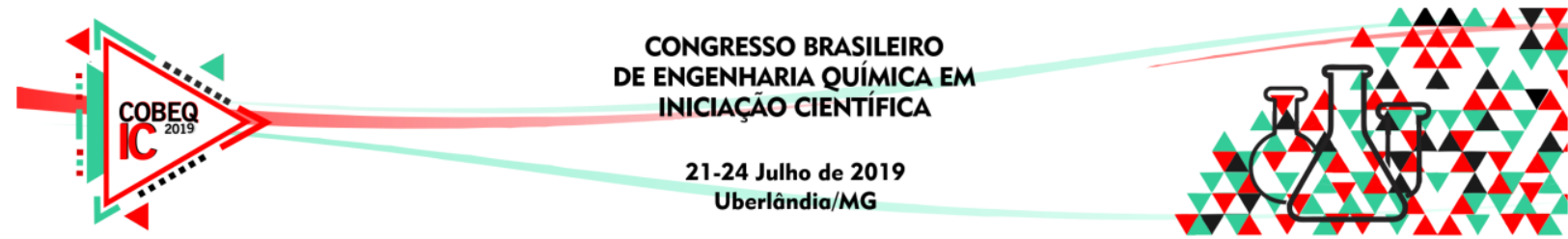

\title{
ANÁLISE DE CORRELAÇÕES DA DIFUSIVIDADE EFETIVA DE GRÃOS DE SOJA SUBMETIDOS A SECAGEM EM CAMADA FINA
}

\author{
F. D'AVILA ${ }^{1}$, R. M. URNAU ${ }^{1}$, A. M. ZANELLA ${ }^{1}$ e M. F. P. MOREIRA ${ }^{1}$ \\ ${ }^{1}$ Universidade Estadual do Oeste do Paraná, Departamento de Engenharia Química \\ Centro de Engenharias e Ciências Exatas \\ E-mail para contato: felipegdavila@gmail.com
}

\begin{abstract}
RESUMO - A soja é uma das principais lavouras do mundo e a operação de secagem desempenha importante etapa em seu beneficiamento. $\mathrm{O}$ presente estudo teve como objetivo avaliar modelos para previsão da difusividade efetiva da soja, parâmetro este importante nos estudos de secagem. Utilizou-se o método das tangentes para a determinação das difusividades da soja em função da umidade do grão e da temperatura do ar de secagem. A correlação proposta por Dotto et al. (2018) foi a que apresentou menor erro médio $(0,048 \%)$ para os dados médios. Para os dados puntuais de difusividade, foram necessárias modificações no modelo, o que proporcionou um erro máximo de $2,6 \%$.
\end{abstract}

\section{INTRODUÇÃO}

Uma cultura de destaque na economia brasileira, a soja (Glycine max L. Merril) é responsável por 28,13\% das exportações do agronegócio nacional (AGROSTAT, 2019). No estado do Paraná, o grão é o produto agrícola mais exportado.

Sendo um grão colido com alto teor de umidade, a secagem é uma operação de grande importância, uma vez que evita a deterioração físico-química e microbiológica, além de garantir a qualidade do produto (Krzyzanowski et al., 2008).

A difusividade efetiva é um coeficiente capaz de descrever a velocidade com que a água é retirada de um material. Este parâmetro representa todos os fenômenos envolvidos na transferência da massa de água (Lewis, 1921).

Compreender os fenômenos que acontecem durante o processo é fundamental no desenvolvimento e otimização de equipamentos, uma vez que a relação usual da difusividade com a equação de Arrhenius não se mostra satisfatória em diversos materiais (Touil et al., 2014; Dotto et al., 2018), o presente estudo teve como objetivo avaliar diferentes modelos para a estimativa deste coeficiente.

\section{MATERIAIS E MÉTODOS}

Para o estudo, utilizaram-se grãos de soja das safras de 2015/2016/2017 da região oeste do Paraná, cedidos pela Coamo Agroindustrial Cooperativa. Os mesmos foram caracterizados quanto ao teor de umidade e dimensões, em um espaço amostral de 30 unidades. 


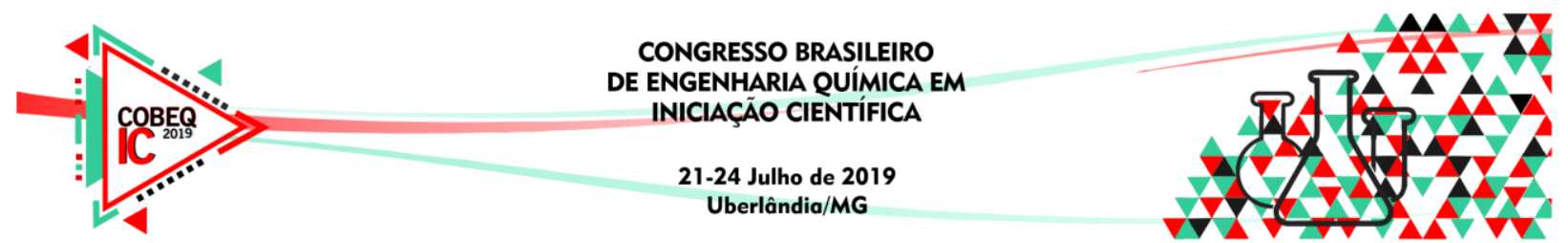

As secagens em camada fina foram realizadas em diferentes condições de temperatura (de 30 a $63{ }^{\circ} \mathrm{C}$ ) e de velocidade do ar (de 0,1 a $1,1 \mathrm{~m} \mathrm{~s}^{-1}$ ). O teor de umidade em base seca das amostras de grãos foi determinado através da metodologia proposta por MAPA (2009), através de secagem em estufa, a $105^{\circ} \mathrm{C}$.

O teor de umidade de equilíbrio dos grãos $\left(\mathrm{X}_{\mathrm{e}}\right)$ foi determinado através da isoterma de equilíbrio Halsey modificada (Osborn et al., 1989), Equação 1, com as constantes empíricas determinadas por Finkler e Moreira (2010).

$$
X_{e}=\left(\frac{\exp (-0,0124 \cdot T+3,2286)}{-\ln (\mathrm{UR})}\right)^{1 / 1,4522}
$$

onde T é a temperatura $\left({ }^{\circ} \mathrm{C}\right)$ e UR é a umidade relativa do ar de secagem em base decimal.

O modelo difusivo da Segunda Lei de Fick, em coordenadas esféricas (Crank, 1975), utilizado para analisar a transferência de massa, desconsiderando resistência externa, está exposto na Equação 2.

$$
\frac{\partial \mathrm{X}}{\partial \mathrm{t}}=\frac{\mathrm{D}_{\mathrm{EF}}}{\mathrm{r}^{2}}\left[\frac{\partial}{\partial \mathrm{r}}\left(\mathrm{r}^{2} \frac{\partial \mathrm{X}}{\partial \mathrm{r}}\right)\right]
$$

Condição inicial

$$
\begin{array}{ll}
\text { Condição inicial } & \mathrm{t}=0, \mathrm{X}=\mathrm{X}_{0} \\
\text { Condição de simetria } & \mathrm{r}=0, \partial \mathrm{X} / \partial \mathrm{r}=0 \\
\text { Condição de contorno } & \mathrm{r}=\mathrm{R}, \mathrm{X}=\mathrm{X}_{\mathrm{e}}
\end{array}
$$

A solução da formulação é representada pela seguinte equação:

$$
\overline{\mathrm{W}}=\frac{\overline{\mathrm{X}}-\mathrm{X}_{\mathrm{e}}}{\mathrm{X}_{0}-\mathrm{X}_{\mathrm{e}}}=\frac{6}{\pi^{2}} \cdot \sum_{\mathrm{n}=1}^{\infty}\left\{\frac{1}{\mathrm{n}^{2}} \cdot \exp \left[\frac{-(\mathrm{n} \cdot \pi)^{2} \cdot \mathrm{D}_{\mathrm{EF}} \cdot \mathrm{t}}{\mathrm{R}^{2}}\right]\right\}
$$

em que $\overline{\mathrm{W}}$ é o adimensional de umidade livre, $\overline{\mathrm{x}}, \mathrm{X}_{\mathrm{e}}$ e $\mathrm{x}_{0}$ são os teores de umidade em base seca em qualquer instante, no equilíbrio e inicial, respectivamente, DeF a difusividade efetiva $\left(\mathrm{m}^{2} \mathrm{~s}^{-1}\right)$, R o raio médio de partícula $(\mathrm{m})$ e t o tempo do processo $(\mathrm{s})$.

A difusividade específica experimental em um determinado instante de tempo foi determinada por meio do método da tangente, com auxílio das seguintes Equações:

$$
\begin{aligned}
& D_{\mathrm{EF}}=\left[\frac{(\mathrm{d} \overline{\mathrm{W}} / \mathrm{dt})_{\text {exp }}}{(\mathrm{d} \overline{\mathrm{W}} / \mathrm{dFo})_{\text {teo }}}\right] \cdot \mathrm{R}^{2} \\
& \left(\frac{\mathrm{d} \overline{\mathrm{W}}}{\mathrm{dFO}}\right)_{\text {teo }}=-6 \cdot \sum_{\mathrm{n}=1}^{\infty}\left\{\exp \left[-(\mathrm{n} \cdot \pi)^{2} \cdot \mathrm{Fo}\right]\right\}
\end{aligned}
$$

onde Fo é o número de Fourier (adimensional).

Modelos de difusividade efetiva disponíveis na literatura e propostos foram ajustados aos valores determinados pelo método da tangente, por meio do software livre Scilab ${ }^{\circledR}$ (www.scilab.org), com os parâmetros de cada equação ajustados pelo método de Levenberg-Marquardt de otimização. 


\section{RESULTADOS E DISCUSSÕES}

\subsection{Caracterização dos grãos de soja}

Os grãos de soja analisados apresentaram um diâmetro médio de 6,572 mm e uma esfericidade média de 0,89 . Os valores corroboram estudos presentes na literatura (Guedes et al., 2011; Bortolotti, 2012). O teor de umidade inicial médio, em base seca, foi de 18,41\%, valor próximo a média do teor em diferentes condições de colheita (Tsukahara et al., 2016).

\subsection{Análise da difusividade efetiva média}

Com a consideração de que a difusividade efetiva é constante durante uma secagem, uma equação do tipo Arrhenius e a cinco diferentes modelos propostos na literatura, apresentados na Tabela 1 , foram ajustados aos valores experimentais médios, determinados pelo método das tangentes.

Tabela 1 - Modelos da difusividade efetiva

\begin{tabular}{|l|c|c|c|}
\hline \multicolumn{1}{|c|}{ Autor } & Modelo & \multicolumn{2}{c|}{ Erro médio (\%) } \\
\cline { 3 - 5 } Arrhenius & $\mathrm{D}_{\mathrm{EF}}(\mathrm{T})=\mathrm{D}_{0} \cdot \exp \left(-\mathrm{E}_{\mathrm{a}} / \mathrm{R}_{\mathrm{g}} \cdot \mathrm{T}\right)$ & $29,36 \%$ & $21,66 \%$ \\
\hline $\begin{array}{l}\text { Chemkhi } \\
\text { Zagrouba, } 2005\end{array}$ & $\mathrm{D}_{\mathrm{EF}}(\mathrm{X})=\mathrm{a} \cdot \mathrm{X}^{2}+\mathrm{b} \cdot \mathrm{X}+\mathrm{c}$ & $38,83 \%$ & $20,30 \%$ \\
\hline Azzouz et al., 2002 & $\mathrm{D}_{\mathrm{EF}}(\mathrm{T}, \mathrm{X})=\mathrm{D}_{0} \cdot \exp \left(-\mathrm{E}_{\mathrm{a}} / \mathrm{R}_{\mathrm{g}} \cdot \mathrm{T}\right) \cdot \exp (-(\mathrm{a} \cdot \mathrm{T}+\mathrm{b}) \cdot \mathrm{X})$ & $28,14 \%$ & $20,90 \%$ \\
\hline Touil et al., 2014 & $\mathrm{D}_{\mathrm{EF}}(\mathrm{T}, \mathrm{X})=\mathrm{a}+\mathrm{b} \cdot \mathrm{X}+\mathrm{c} \cdot \mathrm{T}+\mathrm{d} \cdot \mathrm{X}^{2}+\mathrm{e} \cdot \mathrm{T}^{2}+\mathrm{f} \cdot \mathrm{X} \cdot \mathrm{T}$ & $25,07 \%$ & $7,10 \%$ \\
\hline Barrozo et al., 2006 & $\mathrm{D}_{\mathrm{EF}} / \mathrm{R}^{2}(\mathrm{~T}, \mathrm{X})=\mathrm{a} \cdot \exp \left(-\mathrm{E}_{\mathrm{a}} / \mathrm{R}_{\mathrm{g}} \cdot \mathrm{T}\right) \cdot \exp (\mathrm{b} \cdot \mathrm{X})$ & $35,84 \%$ & $26,20 \%$ \\
\hline Dotto et al., 2018 & $\ln \left(1 / \mathrm{D}_{\mathrm{EF}}\right)=\left(\mathrm{a}_{1} \cdot \mathrm{T}^{\mathrm{a}_{2}}+\mathrm{a}_{3}\right) \cdot \mathrm{W}^{2}+\left(\mathrm{b}_{1} \cdot \mathrm{T}^{\mathrm{b}_{2}}+\mathrm{b}_{3}\right) \cdot \mathrm{W}+\left(\mathrm{c}_{1} \cdot \mathrm{T}^{\mathrm{c}_{2}}+\mathrm{c}_{3}\right)$ & $0,940 \%$ & $0,048 \%$ \\
\hline
\end{tabular}

A equação do tipo Arrhenius apresentou uma baixa correlação, conforme apresentado na Figura 1 (a). Do modelo, pode-se estimar a energia de ativação para os grãos de soja, de aproximadamente $27,77 \mathrm{~kJ} \mathrm{~mol}^{-1}$. Kitic e Viollaz (1984), ao estudarem a secagem de grãos de soja em camada fina, obtiveram uma energia de ativação de $28,80 \mathrm{~kJ} \mathrm{~mol}^{-1}$, corroborando o presente trabalho.

Figura 1 - Ajuste da equação de Arrhenius (a), influência do teor de umidade sobre a difusividade efetiva (b) e correlação entre valores experimentais e calculados de DEF pelo modelo de Dotto para valores médios (c).

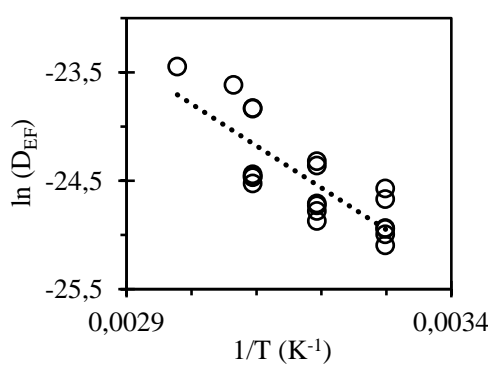

(a)

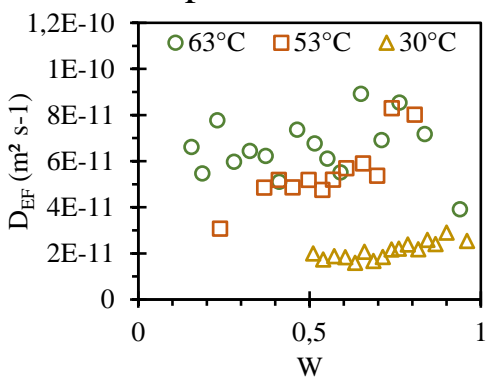

(b)

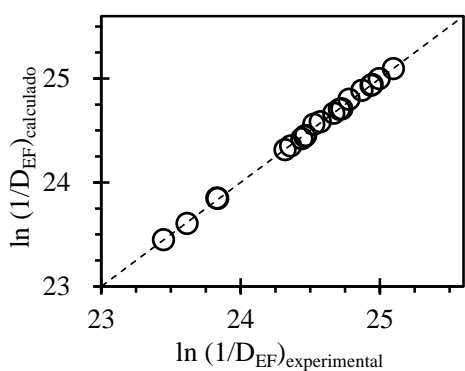

(c) 


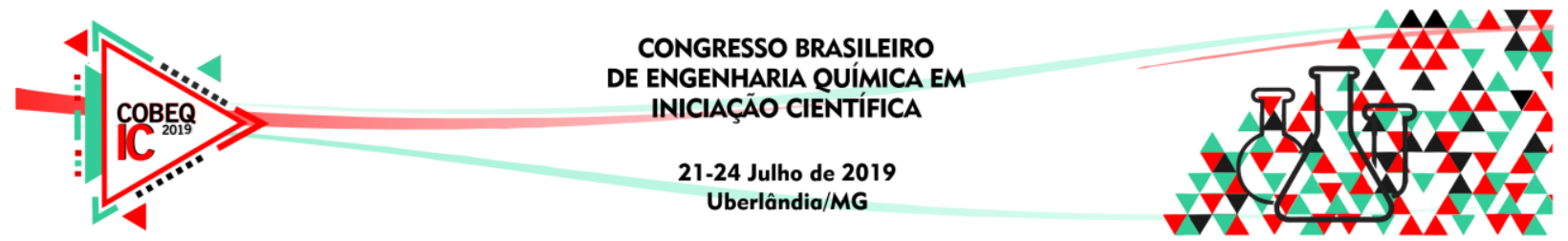

O uso deste tipo de equação, além de considerar a difusividade efetiva como dependente apenas da temperatura do ar, a considera como constante durante todo o processo de secagem (Touil et al., 2014). A Figura 1 (b) expõe a relação entre a difusividade efetiva e o adimensional de umidade, para 3 diferentes temperaturas. Observa-se que a variação do teor de umidade exerce efeito sobre a difusividade, assim como temperatura.

Chemkhi e Zagrouba (2005) constataram que, para materiais argilosos, a difusividade efetiva tem uma relação mais significativa com o teor de umidade do que com a temperatura. Os autores propuseram um modelo que, ao ser ajustado aos dados obtidos com os grãos de soja atingiu um coeficiente de determinação $\left(\mathrm{R}^{2}\right)$ de 0,5076 e erro médio de $20,3 \%$.

Os modelos analisados com dependência de apenas uma variável não proporcionaram uma boa previsão dos dados experimentais. Diferentes trabalhos presentes na literatura estudaram modelos dependentes de temperatura e teor de umidade, como Azzouz et al. (2002), Barrozo et al. (2006), Touil et al. (2014) e Dotto et al. (2018). Os valores para os erros médios percentuais para as equações desenvolvidas por estes autores estão presentes na Tabela 1, na coluna "Médios". Observa-se que, de maneira geral, todos os modelos que levam em conta umidade e temperatura apresentaram erros médios menores do que aqueles que só consideram ou temperatura ou umidade. Dentre os modelos, o de Dotto et al. (2018) apresentou melhor ajuste, proporcionando um desvio médio de 0,048\%, exposto na Figura 1 (c). Os parâmetros obtidos foram $\mathrm{a}_{1}=1,213 \times 10^{-10} \mathrm{~K}^{-1}$, a2 $=4,400$, $\mathrm{a}_{3}=12,805, \mathrm{~b}_{1}=-0,0242 \mathrm{~K}^{-1}, \mathrm{~b}_{2}=1,222, \mathrm{~b}_{3}=0,1371, \mathrm{c}_{1}=-8,261 \times 10^{-22} \mathrm{~K}^{-1}, \mathrm{c}_{2}=8,261 \mathrm{e}$ $\mathrm{c}_{3}=31,654$.

Entretanto, uma vez que durante o processo de secagem o teor de umidade do material varia e, como já definido, a difusividade efetiva depende desta variável, não se pode considerá-la como constante durante a operação. Desta forma, os modelos foram ajustados para a difusividade efetiva puntual, avaliada em cada ponto da cinética de secagem.

\subsection{Análise da difusividade efetiva puntual}

Os erros percentuais médios obtidos para cada equação estão expostos na Tabela 1, na coluna "Puntuais". Assim como para os dados médios, os modelos dependentes tanto da temperatura quanto do teor de umidade mostraram-se mais eficazes. Dentre eles, o modelo de Dotto et al. (2018) novamente apresentou menor desvio.

Em geral, os valores de erro médio obtidos foram significativamente superiores em relação à consideração de difusividades médias. Tal comportamento era esperado, visto o sensível incremento no número de dados utilizados. Enquanto no ajuste dos dados médios foram utilizados 20 dados, no ajuste de dados puntuais utilizou-se um total de 320 pontos. Este é um ponto importante a se atentar nos trabalhos que tratam sobre previsão de difusividade efetiva.

A Tabela 2 apresenta a equação modificada de Dotto et al. (2018), a qual melhor previu os dados experimentais. Os parâmetros obtidos foram $\mathrm{a}_{1}=7,111 \times 10^{-13} \mathrm{~K}^{-1}$, a $2=-974,527$, $\mathrm{b}_{1}=-2,040 \times 10^{-13} \mathrm{~K}^{-1}, \mathrm{~b}_{2}=277,080, \mathrm{c}_{1}=1,882 \times 10^{-9} \mathrm{~K}^{-1} \mathrm{e}_{2}=0,7633$. 


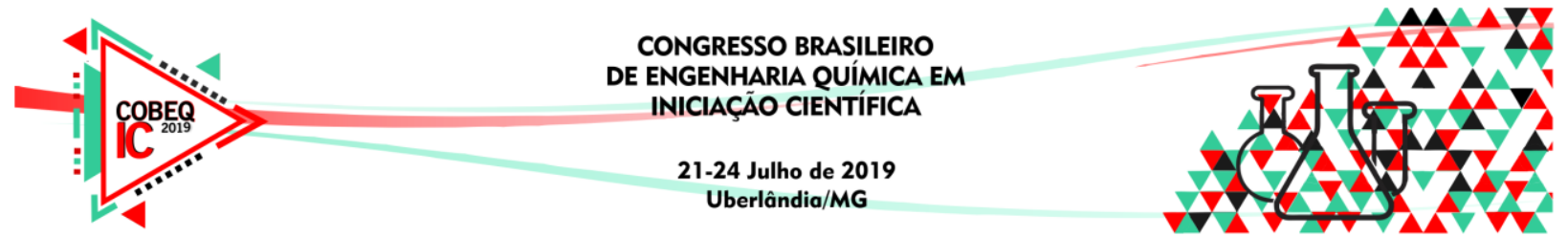

Tabela 2 - Correlação proposto da difusividade efetiva

\begin{tabular}{|c|c|c|c|}
\hline \multirow{2}{*}{ Modelo } & \multicolumn{2}{|c|}{ Erro médio (\%) } & \multirow{2}{*}{ Equação } \\
\cline { 2 - 3 } & Puntuais & Médios & (9) \\
\hline \hline $\ln \left(1 / \mathrm{D}_{\mathrm{EF}}\right)=\left(\mathrm{a}_{1} \cdot \mathrm{T}^{6}+\mathrm{a}_{2}\right) \cdot \mathrm{X}^{2}+\left(\mathrm{b}_{1} \cdot \mathrm{T}^{6}+\mathrm{b}_{2}\right) \cdot \mathrm{X}+\left(\mathrm{c}_{1} \cdot \mathrm{T}^{4}+\mathrm{c}_{2}\right)$ & $0,880 \%$ & $0,147 \%$ & (9) \\
\hline
\end{tabular}

\subsection{Comparação entre as análises}

As correlações com melhor desempenho na análise para difusividade puntual e média, com seus respectivos parâmetros, foram comparadas quanto a capacidade de prever valores para cada instante. A Figura 2 mostra a correlação entre os dados previstos e os dados experimentais. Observa-se que ao utilizar o modelo ajustado aos dados médios os erros aumentam significativamente. Enquanto os erros não superaram 2,6\% utilizando o modelo de dados puntual, o uso do modelo de dados médio apresentou erros na faixa de $-6 \%$ a $19 \%$.

Figura 2 - Correlação entre valores calculados e experimentais de DEF pela consideração de difusividade como variável (a) e como constante (b) ao longo de uma secagem.

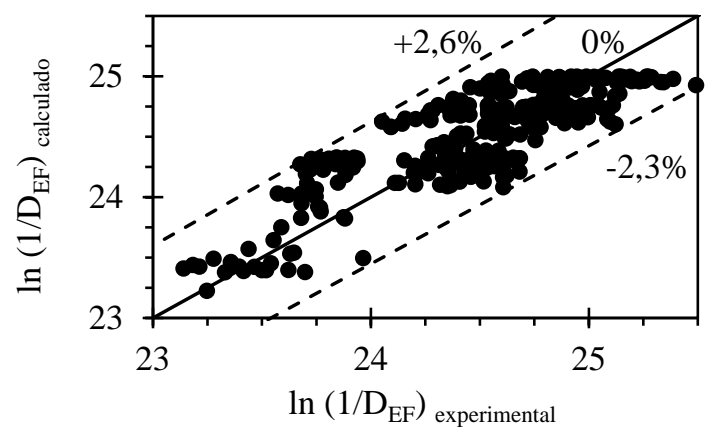

(a)

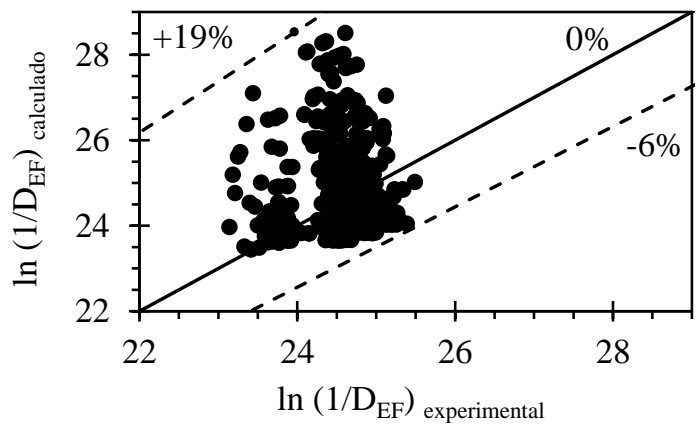

(b)

\section{CONCLUSÃO}

A energia de ativação dos grãos de soja, pela equação de Arrhenius, foi de $27,77 \mathrm{~kJ} \mathrm{~mol}^{-1}$, corroborando a literatura. Comprovou-se a dependência da difusividade efetiva com a temperatura e com o teor de umidade. O modelo de Dotto et al. (2018) propiciou melhor ajuste aos dados médios (erro médio de 0,048\%), considerando a difusividade como constante durante o processo de secagem. Entretanto, a modificação no modelo de Dotto proposta neste trabalho permitiu uma previsão dos valores de difusividade a cada ponto de um processo de secagem com um erro máximo de $2,6 \%$.

\section{AGRADECIMENTOS}

Os autores agradecem à Coamo Agroindustrial Cooperativa pelo fornecimento dos grãos de soja para a realização do estudo.

\section{REFERÊNCIAS}

AGROSTAT. Estatística de Comércio Exterior do Agronegócio Brasileiro. Ministério da Agricultura, Pecuária e Abastecimento, 2019. 


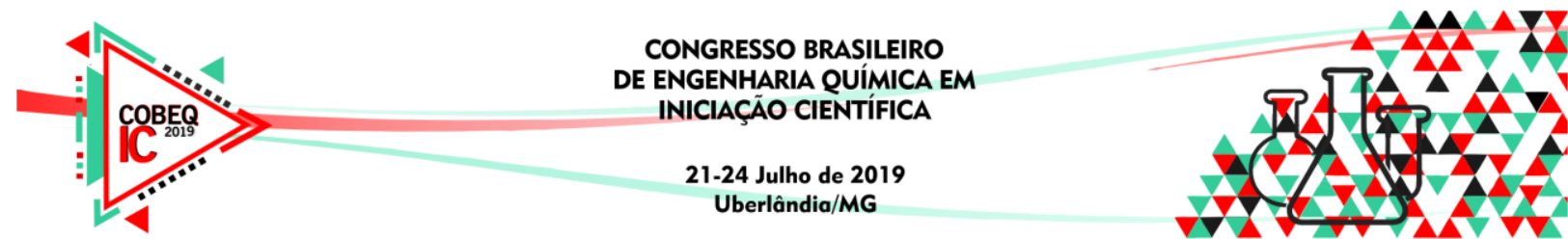

AZZOUZ, S.; GUIZANI, A.; JOMAA, W.; BELGHITH, A. Moisture diffusivity and drying kinetic equation of convective drying of grapes. J. Food Eng., v. 55, p. 323-330, 2002.

BARROZO, M. A. S.; HENRIQUE, H. M.; SARTORI, D. J. M.; FREIRE, J. T. The use of the orthogonal collocation method on the study of the drying kinetics of soybean seeds. J. Sto. Prod. Res., v. 42, p. 348-356, 2006.

BORTOLOTTI, C. T. Estudo experimental da fluidodinâmica de uma mistura de resíduo de acerola e soja em leito de jorro. Dissertação de Mestrado - UFU, p. 1-120, 2012.

CHEMKHI, S.; ZAGROUBA, F. Water diffusion coefficient in clay material from drying data. Desalination, v.185, p. 491-198, 2005.

CRANK, J. The Mathematics of Diffusion. London: Clarendon Press, 1975.

DOtTO, G. L.; MeIli, L.; TANABE, E. H.; CHIElle, D. P.; MOREIRA, M. F. P. Evaluation of the mass transfer process on thin layer drying of papaya seeds from the perspective of diffusive models. Heat Mass Transf., v. 54, p. 463-471, 2018.

DOYMAZ, I. Drying behaviour of green beans. J. Food Eng., v. 69, p. 161-165, 2005.

FINKLER, A. T. J.; MOREIRA, M. F. P. Análise das isotermas de equilíbrio de adsorção e dessorção da soja. Foz do Iguaçu: Anais do XVIII Congresso Brasileiro de Engenharia Química, 2010.

GUEDES, M. A.; MATA, M. E. R. M. C.; DUARTE, M. E. M.; FARIAS, P. A. Caracterização física de grãos de soja utilizando-se processamento digital de imagens. Rev. Bra. de Prod. Agroind., v. 13, n. 3, p. 279-294, 2011.

KRZYZANOWSKI, F. C.; FRANÇA NETO, J. B.; HENNING, A. A.; COSTG, N. P. $O$ controle de qualidade agregando valor à semente de soja - série sementes. Londrina: Embrapa Soja, 2008.

LEWIS, W. K. The rate of drying of solids materials, The J. of Ind. and Eng. Chem., v. 13, n. 5 , p. 427-432, 1921.

MAPA. Regras para Análise de Sementes. Brasil: Ministério da Agricultura, Pecuária e Abastecimento, 2009.

OSBORN, G.S.; WHITE, G.M.; SULAIMAN, A.H.; WELTON, L.R. Predicting equilibrium moisture proportions of soybeans. Transac. of the ASAE, v. 32(6) p. 2109-2113, 1989.

TOUIL, A.; CHEMKHI, S.; ZAGROUBA, F. Moisture Diffusivity and Shrinkage of Fruit and Cladode of Opuntia ficus-indica during Infrared Drying. Jour. of Food Proc., v. 2014, p. 1-9, 2014.

TSUKAHARA, R. Y.; FONSECA, I. C. B.; SILVA, M. A. A.; KOCHINSKI, E. G.; NETO, J. P.; SUYAMA, J. T. Produtividade de soja em consequência do atraso da colheita e de condições ambientais. Pesq. agropec. bras. v. 51, n. 8, p. 905-915, 2016. 